\title{
The Construct Validity of the Dutch Personality Inventory for DSM-5 Personality Disorders (PID-5) in a Clinical Sample
}

Assessment

20I6, Vol. 23(I) 42-5I

(C) The Author(s) 2015

Reprints and permissions:

sagepub.com/journalsPermissions.nav

DOI: 10.1 I77/I073191।I5575069

asm.sagepub.com

\author{
Tim Bastiaens', Laurence Claes ${ }^{2}$, Dirk Smits ${ }^{2,3}$, Barbara De Clercq ${ }^{4}$, \\ Filip De Fruyt ${ }^{4}$, Gina Rossi ${ }^{5}$, Dominique Vanwalleghem', Rudi Vermote ${ }^{1,2}$, \\ Benedicte Lowyck ${ }^{1,2}$, Stephan Claes ${ }^{1,2}$, and Marc De Hert ${ }^{1,2}$
}

\begin{abstract}
The factor structure and the convergent validity of the Personality Inventory for DSM-5 (PID-5), a self-report questionnaire designed to measure personality pathology as advocated in the fifth edition, Section III of Diagnostic and Statistical Manual of Mental Disorders (DSM-5), are already demonstrated in general population samples, but need replication in clinical samples. In 240 Flemish inpatients, we examined the factor structure of the PID-5 by means of exploratory structural equation modeling. Additionally, we investigated differences in PID-5 higher order domain scores according to gender, age and educational level, and explored convergent and discriminant validity by relating the PID- 5 with the Dimensional Assessment of Personality Pathology-Basic Questionnaire and by comparing PID-5 scores of inpatients with and without a DSM-IV categorical personality disorder diagnosis. Our results confirmed the original five-factor structure of the PID-5. The reliability and the convergent and discriminant validity of the PID-5 proved to be adequate. Implications for future research are discussed.
\end{abstract}

\section{Keywords}

PID-5, personality disorders, personality traits, DSM-5, assessment

As the traditional categorical paradigm for personality disorders described in the fourth edition of the Diagnostic and Statistical Manual of Mental Disorders (DSM-IV; American Psychiatric Association [APA], 2000) has become subject to a number of conceptual and psychometrical criticisms (e.g., Ryder, Costa, \& Bagby, 2007; Trull \& Durrett, 2005), various dimensional models of personality pathology have been proposed as a valuable alternative. The Personality Inventory for DSM-5 (PID-5; Krueger, Derringer, Markon, Watson, \& Skodol, 2012) is a self-report questionnaire developed to help clinicians assess personality traits in line with the dimensional perspective of personality pathology as advocated in Section III of DSM-5 (APA, 2013). The process of its development started from a review of existing models and measures of maladaptive personality traits, with a particular interest in the work of Widiger and Simonsen (2005), who conceptualized four bipolar domains able to account for the findings of no less than 18 personality trait models in the literature. These four bipolar domains are (a) Extraversion versus Introversion, (b) Antagonism versus Compliance, (c) Constraint versus Impulsivity, and (d) Negative Affect versus Emotional Stability. In addition to these four bipolar domains, Widiger and Simonsen (2005) also included a fifth domain named Unconventionality (or Openness) versus Closedness to Experience, articulated as a separate entity in 3 of the 18 models reviewed, and referring to key aspects of schizotypy (see e.g., Chmielewski \& Watson, 2008; Harkness, McNulty, \& Ben-Porath, 1995). Starting from a hypothesized set of domains covering existing models, work group members generated an initial list of 37 specific personality traits, translated these into items tapping into the trait content, and scrutinized the emerging factor structure. This process resulted in a final set of 27 primary facet scales that have been reported to group into five or six pathological higher order domains. The

\footnotetext{
'University Psychiatric Centre, KU Leuven, Campus Kortenberg, Kortenberg, Belgium

${ }^{2} \mathrm{KU}$ Leuven, Leuven, Belgium

${ }^{3}$ Odisee University College, Brussels, Belgium

${ }^{4}$ Ghent University, Ghent, Belgium

${ }^{5}$ Vrije Universiteit Brussel, Brussels, Belgium
}

\section{Corresponding Author:}

Tim Bastiaens, University Psychiatric Centre, KU Leuven, Campus Kortenberg, Leuvensesteenweg 517, 3070 Kortenberg, Belgium. Email: tim.bastiaens@uc-kortenberg.be 
five-factor solution parallels the nonclinical five-factor model (Costa \& McCrae, 1992; see the appendix) and is composed of the higher order domains (a) Negative Affectivity, (b) Detachment, (c) Antagonism, (d) Disinhibition, and (e) Psychoticism (Krueger et al., 2012).

Since the original article by Krueger et al. (2012), sampling treatment seekers, a considerable number of studies have provided evidence for (a) the factor structure and (b) the convergent validity of the PID-5 in undergraduate and general population samples. Wright et al. (2012), for example, replicated the original PID-5 factor structure in a sample of college students, and framed it in a hierarchy of one, two, three, four, and five factors, respectively. Using an Italian nonclinical sample, Fossati, Krueger, Markon, Borroni, and Maffei (2013) corroborated the PID-5 factor structure as well, and investigated its associations with the Personality Diagnostic Questionnaire-4+ and with a measure of psychopathy. In undergraduate students, Hopwood, Thomas, Markon, Wright, and Krueger (2012) related the PID-5 primary facet scales and higher order domains to the six $D S M-I V$ personality disorders the $D S M-5$ Task Force proposed to retain as categorical entities, as well as to the six (i.e., including the passive-aggressive and the depressive from the appendices) personality disorders proposed to be removed. Samuel, Hopwood, Krueger, Thomas, and Ruggero (2013) performed a similar study in a college sample, using PID-5 primary facet scale scores to reproduce $D S M-I V$ personality disorders. In undergraduates, Anderson et al. (2013) provided empirical evidence for the hypothesized relations between each PID-5 higher order domain and its conceptually related Personality Pathology Five variant (Harkness, Finn, McNulty, \& Shields, 2012; Harkness, McNulty, \& Ben-Porath, 1995). Other research in nonclinical samples has related the PID-5 higher order domains to the normal range five-factor model instruments (e.g., De Fruyt et al., 2013; Gore \& Widiger, 2013; Thomas et al., 2012; Wright \& Simms, 2014).

To date, however, the number of studies on (a) the factor structure and (b) the convergent and discriminant validity of the PID-5 in clinical samples is fairly limited. An impressive study by Few et al. (2013) in an outpatient clinical sample investigated the relation between PID-5 scores and a number of self-report scales as well as clinician-rated $D S M-I V$ Axis II personality disorders, but did not include a separate investigation of the PID-5 factor structure. The same is true for the outpatient study by Watson, Stasik, Ro, and Clark (2013), who examined the PID-5 in relation to the Big-Three (Negative Temperament, Positive Temperament, and Disinhibition) and the Big-Five model, but did not report findings on the PID-5 factor structure itself. As clinical samples can differ from nonclinical samples regarding prevalence, form, and/or severity of personality pathology, it is plausible that the distribution of PID-5 traits in clinical samples is different from the distribution of traits in nonclinical samples. To our knowledge, only one study investigated the PID-5 higher order domain structure in a German sample of 577 students and 212 psychiatric inpatients (Zimmerman et al., 2014). Whereas the structure did replicate in the college sample, the factor congruence in the clinical sample was troubled by failure to replicate the Disinhibition factor. In the APA field trial as reported by Quilty, Ayearst, Chmielewski, Pollock, and Bagby (2013), only the unidimensionality of the PID-5 scales was investigated in a sample of 201 psychiatric patients.

Since the PID-5 is conceptually developed to capture personality pathology, evidence on its factor structure in nonclinical samples needs to be supplemented with factor replication and more elaborate validation studies in a variety of clinical samples (De Fruyt et al., 2013; Hopwood et al., 2012; Thomas et al., 2012; Wright et al., 2012). Thus far, confirmation of the original five-factor structure of the Dutch version of the PID-5 was performed (only) in a sample of undergraduate psychology students (De Fruyt et al., 2013). Hence, the first aim of the current study was to investigate whether the factor structure of the Dutch PID-5 can be replicated in a sample of clinical inpatients.

As a second aim, we explored possible differences in PID-5 higher order domain scores according to gender, age, and years of education. Regarding age, earlier findings by Van den Broeck, Bastiaansen, Rossi, Dierckx, and De Clercq (2013) in a nonclinical sample revealed significant betweengroup differences for facets of Antagonism and Disinhibition, with younger adults (age 17-40 years) always scoring higher than older adults (age 61-99 years). Regarding gender and years of education, our approach was exploratory.

The third aim was to explore the relationship between the PID-5 higher order structure and another dimensional model of maladaptive personality traits that closely resembles the theoretical base of the PID-5, that is, the Dimensional Assessment of Personality Pathology (DAPP) model as measured by the Dimensional Assessment of Personality Pathology—Basic Questionnaire (DAPP-BQ; Livesley \& Jackson, 2009; Dutch translation: Van Kampen $\&$ de Beurs, 2009). The DAPP-BQ has already demonstrated meaningful relations with other dimensional personality instruments (e.g., Krueger \& Tackett, 2003; Krueger et al., 2011) and its relationship with the PID-5 has already been successfully documented in older general population adults (Van den Broeck, Bastiaansen, Rossi, Dierckx, De Clercq, and Hofmans, 2013). In terms of alignment, we hypothesized that each PID-5 higher order domain would correlate the highest with its DAPP-BQ higher order dimension counterpart. Next to an investigation of alignment (or convergence), we also explored possible differences between both models. That is, next to the articulation of a fifth domain Psychoticism in the PID-5 model, not present in the DAPP-BQ model, domains that essentially try to capture the same general construct can still differ in broadness or might still tap into slightly 
different contents, for example, because of the way they are keyed (i.e., positive or negative).

As a fourth and final aim, we explored possible differences in PID-5 higher order domain scores according to the presence versus absence of $D S M-I V$ personality disorder diagnosis.

\section{Method}

\section{Participants and Procedure}

All consecutive admitted inpatients of different sections of the University Psychiatric Centre, KU Leuven Campus, Kortenberg (Belgium) were invited to participate in this study by the psychologist who conducted the general assessment for clinical purposes on intake. A total number of 257 inpatients were initially included in this study. Of the total group, 240 participants returned data without missing responses. This subsample was retained for the current analyses. Patient's age, gender, and level of education were based on the electronic patient files of the hospital. One hundred sixteen respondents were female (48.3\%) and 123 were male $(51.3 \%)$, with gender information missing for one respondent. The mean age was $33.0(S D=11.2$; range 17-65 years). The level of education was coded in terms of the minimal number of years needed to complete a level. ${ }^{1}$ Conceptualized this way, the mean level of education was 12.6 years $(S D=2.6$; range 6-16.5 years, modus $=$ degree after 6 years of secondary school, 48.9\%). Individual $D S M-I V$ primary diagnoses were assessed by means of a clinical interview by a licensed psychiatrist and retrieved through the electronic patient files of the hospital. To determine personality disorder diagnoses the Structured Clinical Interview for $D S M-I V$ Personality Disorders (SCID-II; First, Gibbon, Spitzer, Williams, \& Benjamin, 1997) was conducted. Primary DSM-IV diagnoses for the total group are depicted in Table 1. All patients filled in the questionnaires after providing written informed consent. The study was approved by the medical ethical committee of the University Psychiatric Centre, KU Leuven Campus, Kortenberg.

\section{Instruments}

The PID-5 (Krueger et al., 2012; Dutch translation: De Clercq, De Fruyt, Mervielde, Krueger, \& Markon, 2011) is a self-report questionnaire that consists of 220 items scored on a 4-point Likert-type scale ranging from 0 (not at all true) to 3 (very true). The 220 items group into 25 primary facet scales. In its original format, the 25 primary facet scales are empirically clustered into five higher order domains: (a) Negative Affectivity (b) Detachment, (c) Antagonism, (d) Disinhibition, and (e) Psychoticism.

The DAPP-BQ (Livesley \& Jackson, 2009; Dutch translation: van Kampen $\&$ de Beurs, 2009) is a self-report
Table I. DSM-IV Primary Diagnoses for the Total Group $(N=240)$.

\begin{tabular}{lr}
\hline Primary diagnosis & $n$ \\
\hline Alcohol dependence & 18 \\
Anxiety disorder & $1 \mathrm{I}$ \\
Major depressive disorder & 32 \\
Bipolar disorder & 13 \\
Psychotic disorder & $1 \mathrm{I}$ \\
Personality disorder & 80 \\
Paranoid & I \\
Histrionic & 1 \\
Borderline & 29 \\
Narcissistic & $1 \mathrm{I}$ \\
Antisocial & 2 \\
Dependent & 3 \\
Avoidant & 2 \\
Not otherwise specified & $3 \mathrm{I}$ \\
Adjustment disorder & $1 \mathrm{I}$ \\
Pervasive developmental disorder & 7 \\
Other & $3 \mathrm{I}$ \\
\hline
\end{tabular}

Note. DSM-IV = Diagnostic and Statistical Manual of Mental Disorders-Fourth edition.

questionnaire that consists of 290 items scored on a 5-point Likert-type scale ranging from 1 (very unlike me) to 5 (very like me). The 290 items group into 18 lower order traits that represent specific personality traits. The 18 lower order traits are structured in four higher order dimensions, labeled as Emotional Dysregulation, Inhibitedness, Dissocial Behavior, and Compulsivity (see the appendix).

\section{Analysis}

Factor analyses were performed with Mplus 7.2 (Muthén \& Muthén, 1998-2012). As primary facet scores were used as indicators for the five higher order domains, we first investigated whether the facets themselves were unidimensional (Bandalos, 2002, 2008). As our indicators for the facet factors are the categorical responses to the individual items, model parameters were estimated with the WLSMV algorithm (see e.g., Beauducel \& Yorck-Herzberg, 2006). Subsequently, to investigate the structure of the facet factors in relation to the five higher order domains, we opted for an exploratory structural equation model (Asparouhov $\&$ Muthén, 2009), in which five factors are retained and via a target rotation are rotated toward the solution as found in Krueger et al. (2012). The current approach was preferred to a more restricted confirmatory factor analysis model because of the high number of cross-loadings in the original solution. Model parameters are estimated with maximum likelihood with robust standard errors.

To evaluate model fit, two criteria were used $(\mathrm{Hu} \&$ Bentler, 1998): the comparative fit index (CFI) for fit relative 
to a null model, with values above .95 referring to good model fit and between .90 and .95 to acceptable fit (Hu \& Bentler, 1999), and the standardized root mean square residual (SRMR) as the standardized difference between the observed and the predicted correlation, for which values below .10 refer to acceptable model fit (Kline, 2005). To compare our factor solution with the original model of Krueger et al. (2012), factor congruence coefficients were calculated.

Higher order domain score differences according to gender were investigated by means of independent $t$ tests. Cohen's $d$ was used as a measure of effect size, with a value of .20 denoting a small, .50 a medium, and .80 a large effect. Higher order domain score differences according to age and years of education were investigated by calculating their correlations with PID-5 higher order domain scores, with a value of .10 denoting a small, a value of .30 a medium, and .50 a large effect size.

Convergent and discriminant validity was investigated by correlating the PID-5 higher order factor scores with the DAPP-BQ higher order dimensions. Additionally, to investigate the total variance in each PID-5 higher order domain that can be explained by the DAPP-BQ higher order dimensions, we performed five separate regression analyses, using all DAPP-BQ higher order dimensions as predictors for each PID-5 higher order domain. Possible differences in PID-5 higher order domain scores between patients with and patients without a personality disorder diagnosis were investigated by means of a multivariate analysis of covariance, with diagnosis (personality disorder vs. other) as the independent variable and controlling for gender, age, and level of education. Wilks' lambda was used to determine the significance of the model. Partial eta squared was used as a measure of effect size, with a value of .01 denoting a small, .06 a medium, and .13 a large effect.

\section{Results}

\section{Structure of the PID-5}

Item-facet fit proved satisfactory $(\mathrm{CFI}>.90, \mathrm{SRMR}<.10)$, except for the Facets Hostility and Emotional Lability. Allowing for an error covariance between Item 62 and Item 122 (both referring to fluctuations in emotional intensity, whereas the remaining items refer to variation in emotions), fit for Emotional Lability became acceptable according to CFI (.91), but not satisfactory according to the SRMR (.13). For Hostility CFI was slightly too low (.89) to reach acceptable fit, but SRMR was acceptable (.09). For the higher order domain scales, the fit of the exploratory structural equation modeling with five factors was nearly acceptable $\left(d f=185, \chi^{2}=666.06, \mathrm{CFI}=.86, \mathrm{SRMR}=.04\right)$. Based on the modification indices, an error covariance was allowed between the facets Impulsivity and Grandiosity $(r=-.39)$ resulting in an acceptable model fit $\left(d f=184, \chi^{2}=512.01\right.$, $\mathrm{CFI}=.90, \mathrm{SRMR}=.03)$.
Standardized factor loadings of PID-5 primary facet scales on higher order domains are provided in Table 2 . Comparing our solution with the original model of Krueger et al. (2012), factor congruence coefficients equaled .89 for Negative Affect, .95 for Detachment, .95 for Antagonism, .86 for Disinhibition, and .97 for Psychoticism.

\section{Convergent and Discriminant Validity}

Gender showed a strong effect on Negative Affectivity, with females scoring significantly higher than males $(t(267)=$ $-6.53, p<.001$; Cohen's $d=.80$ ), and a medium effect on Antagonism, with males scoring significantly higher than females $(t(267)=5.41, p<.001$; Cohen's $d=.46)$. Additionally, gender also showed a small effect on Disinhibition $(t(267)=-2.11, p=.04$; Cohen's $d=.26)$, with females scoring significantly higher than males. No significant gender differences were found for Detachment $(t(267)=$ $1.45, p=.15)$ and Psychoticism $(t(267)=-1.43, p=.15)$. Regarding age, a significant negative relation was found with Antagonism $(r=-.15, p=.02)$, Disinhibition $(r=-.28, p<$ $.001)$, and Psychoticism $(r=-.12, p=.002)$. The number of years of education was negatively and significantly related to Disinhibition $(r=-.22, p<.001)$.

The correlations between PID-5 and DAPP-BQ scale scores are shown in Table 3. First, DAPP-BQ Emotional Dysregulation correlated significantly with all PID-5 higher order domains, except for Antagonism. In contrast, PID-5 Negative Affectivity related to DAPP-BQ Emotional Dysregulation much more specifically. Second, except for PID-5 Disinhibition, relating more to DAPP-BQ Dissocial and DAPP-BQ Emotional Dysregulation than (inversely) to DAPP-BQ Compulsivity, each PID-5 higher order domain correlated the highest with its theoretical DAPP-BQ counterpart as expected. Third, PID-5 Psychoticism, not separately represented in the DAPP-BQ, correlated substantially with DAPP-BQ Emotional Dysregulation, DAPP-BQ Dissocial, and DAPP-BQ Inhibitedness. The percentage of variance explained by the total of the four DAPP-BQ higher order dimensions equaled $R^{2}=.73$ for Negative Affectivity, $R^{2}=.59$ for Detachment, $R^{2}=.58$ for Antagonism, $R^{2}=.49$ for Disinhibition, and $R^{2}=.37$ for Psychoticism.

Controlling for gender, age, and years of education, the multivariate analysis of covariance revealed a strong main effect of personality disorder diagnosis, Wilks' lambda $=$ $.82, F(5,206)=9,14, p<.001$, partial $\eta^{2}=.18$, with patients diagnosed with personality disorder displaying the highest scores on the PID-5 higher order factors Negative Affectivity, Antagonism, and Disinhibition in comparison with other patients (see Table 4).

\section{Discussion}

The results of the current study contribute to the ongoing research on personality pathology in different ways. As a 
Table 2. Standardized Factor Loadings of PID-5 Primary Facet Scales on Higher Order Domains.

\begin{tabular}{|c|c|c|c|c|c|}
\hline & Negative Affectivity & Detachment & Antagonism & Disinhibition & Psychoticism \\
\hline Anhedonia & .49 & .67 & .01 & .18 & .04 \\
\hline Anxiousness & .72 & .27 & .01 & .18 & .20 \\
\hline Depressivity & .58 & $.5 \mathrm{I}$ & -.10 & .42 & .04 \\
\hline Emotional Lability & .62 & .04 & .03 & .32 & .13 \\
\hline Hostility & .33 & .25 & .58 & .27 & .07 \\
\hline Perseveration & .43 & .16 & .12 & .17 & .34 \\
\hline Rigid Perfectionism & .39 & .13 & .16 & -.06 & .20 \\
\hline Separation Insecurity & .64 & -.11 & .22 & .15 & .12 \\
\hline Submissiveness & .40 & .14 & -.13 & .17 & .11 \\
\hline Suspiciousness & .33 & .33 & .14 & .24 & .32 \\
\hline Withdrawal & .06 & .79 & .02 & .02 & .20 \\
\hline Attention Seeking & .24 & -.15 & .59 & .25 & .14 \\
\hline Callousness & .03 & .50 & .66 & .16 & .10 \\
\hline Deceitfulness & .10 & .26 & .67 & .41 & .07 \\
\hline Grandiosity & .13 & .09 & .74 & -.23 & .26 \\
\hline Manipulativeness & .11 & .03 & .72 & .28 & .00 \\
\hline Intimacy Avoidance & -.05 & .64 & -.07 & .07 & .18 \\
\hline Restricted Affectivity & -.15 & .57 & .22 & .13 & .18 \\
\hline Distractibility & .33 & .18 & -.07 & .43 & .32 \\
\hline Eccentricity & .13 & .19 & .10 & .30 & .57 \\
\hline Perceptual Dysregulation & .19 & .14 & -.01 & .22 & .73 \\
\hline Risk Taking & -.19 & -.11 & .20 & .53 & .23 \\
\hline Unusual Beliefs and Experiences & .04 & -.02 & .09 & -.03 & .80 \\
\hline Impulsivity & .13 & -.01 & .09 & .68 & .22 \\
\hline Irresponsibility & .07 & .25 & .32 & .56 & .23 \\
\hline \multicolumn{6}{|l|}{ Factor intercorrelations } \\
\hline Negative Affectivity & 1.00 & & & & \\
\hline Detachment & -.08 & 1.00 & & & \\
\hline Antagonism & -.18 & -.07 & 1.00 & & \\
\hline Disinhibition & .12 & -.11 & .10 & 1.00 & \\
\hline Psychoticism & .16 & .08 & .18 & .17 & 1.00 \\
\hline
\end{tabular}

Note. PID-5 = Personality Inventory for DSM-5. Factor loadings above .30 are boldfaced.

Table 3. Correlations Between PID-5 Higher Order Domains and DAPP-BQ Higher Order Dimensions.

\begin{tabular}{|c|c|c|c|c|c|}
\hline \multirow{2}{*}{$\begin{array}{l}\text { DAPP-BQ higher } \\
\text { order dimension }\end{array}$} & \multicolumn{5}{|c|}{ PID-5 higher order domain } \\
\hline & Negative Affectivity & Detachment & Antagonism & Disinhibition & Psychoticism \\
\hline $\begin{array}{l}\text { Emotional } \\
\text { Dysregulation }\end{array}$ & $.72 * *$ & $.23 * *$ & .04 & $.49 * *$ & $.55^{* *}$ \\
\hline Inhibitedness & -.02 & $.74 * *$ & -.14 & .06 & $.25^{*}$ \\
\hline Dissocial & -.01 & .05 & $.72 * *$ & $.56 * *$ & $.45^{* *}$ \\
\hline Compulsivity & $.17 * *$ & -.01 & -.04 & $-.30 * *$ & .07 \\
\hline
\end{tabular}

$* * p<.01 . * p<.05$.

first major finding, the original five-factor structure of PID-5 as reported by Krueger et al. (2012) can be replicated in a clinical sample of Flemish psychiatric inpatients. The factor replication in a clinical sample represents an important next step in the validation process articulated by several authors (e.g., De Fruyt et al., 2013; Hopwood et al., 2012; Wright et al., 2012). As far as we know, only one study of
Zimmerman et al. (2014) has investigated the PID-5 facetlevel factor structure in a sample that included (German) psychiatric patients (as well as college students). Contrary to the German study that experienced problems with the fit of the Disinhibition factor in the clinical subsample, we were able to replicate the original PID-5 factor structure. Factor congruence coefficients, comparing our solution 
Table 4. PID-5 Higher Order Factor Score Means and Standard Deviations for Patients Diagnosed With DSM-IV Personality Disorder Versus Other Pathology, Controlling for Gender, Age, and Years of Education.

\begin{tabular}{|c|c|c|c|c|c|c|}
\hline & \multicolumn{2}{|c|}{ Other } & \multicolumn{2}{|c|}{ Personality disorder } & \multirow[b]{2}{*}{$F$} & \multirow[b]{2}{*}{ Partial $\eta^{2}$} \\
\hline & $M$ & $S D$ & M & $S D$ & & \\
\hline Negative Affectivity & -0.38 & 0.87 & 0.21 & 0.87 & $17.80 * *$ & .08 \\
\hline Detachment & -0.07 & 0.97 & -0.01 & 0.95 & 0.49 & \\
\hline Antagonism & 0.01 & 0.82 & 0.22 & 1.09 & $5.78 * *$ & .03 \\
\hline Disinhibition & -0.25 & 0.75 & 0.29 & 0.99 & $23.55^{* *}$ & .10 \\
\hline Psychoticism & -0.11 & 0.84 & 0.08 & 0.93 & 2.59 & \\
\hline
\end{tabular}

Note. DSM-IV = Diagnostic and Statistical Manual of Mental Disorders-Fourth edition; PID- $5=$ Personality Inventory for DSM-5. Partial $\eta^{2}=.0 \mathrm{I}$ denotes a small effect, partial $\eta^{2}=.06$ a medium effect, and partial $\eta^{2}=.13$ a large effect. $* * p<.01 . * p<.05$.

with the original structure reported by Krueger et al. (2012) were adequate. Contrary to our expectations, we found PID-5 higher order domain intercorrelations in our clinical sample to be lower rather than higher compared with the nonclinical sample of Krueger et al. (2012).

As a second contribution to existing research, our study is the first to preliminary explore PID-5 higher order domain score differences according to gender, age, and years of education in a clinical sample. The strong gender differences in Negative Affectivity found in our inpatient study (with males scoring lower than females) are in line with earlier findings on gender differences in Neuroticism within clinical (e.g., McLean \& Anderson, 2009) as well as general population samples (e.g., Costa, Terracciano, \& McCrae, 2001). In line with general population findings on gender differences in Agreeableness (Costa et al., 2001), showing females to be more agreeable than men, a medium effect of gender regarding Antagonism (i.e., negatively keyed Agreeableness) was found (with males in our sample exhibiting higher mean scores on Antagonism compared with females). No gender difference was observed regarding PID-5 Detachment, whose (reversely keyed) nonclinical analogue Extraversion is described by Costa et al. (2001) as a mixed construct, combining typically male (i.e., dominance) and typically female (i.e., love) components. The albeit small gender effect regarding Disinhibition, showing higher scores of females compared with males, is at odds with reports of higher scores on Conscientiousness in females (Costa et al., 2001), nor can it be explained by the apparent composite structure of the higher order domain Disinhibition discussed below (that is, its relation to the DAPP-BQ higher order dimension Dissocial, representing typically more male Disagreeableness). Our finding is perhaps partly due to the relatively pronounced relation of Disinhibition with the DAPP-BQ higher order dimension Emotional Dysregulation (representing typically more female Neuroticism) as well. Both the decline in Antagonism with increasing age and the absence of a significant association between Negative Affectivity and age parallel earlier findings in nonclinical samples. For example, in two large crosssectional population studies, Donnellan and Lucas (2008) described higher levels of Agreeableness at older age and only a minor association between Neuroticism and age. The inverted relation between age and levels of Antagonism is in line with earlier PID-5 findings by Van den Broeck, Bastiaansen, Rossi, Dierckx, and De Clercq (2013) in a nonclinical sample. Regarding Disinhibition, the expected inverted relation with age was also found. Additionally, a small but significant negative relation was found between age and Psychoticism. The number of years of education was negatively and significantly related to Disinhibition. Importantly, we should take into account that our sample size is only modest in comparison with the population samples used by Costa et al. (2001) and Schmitt, Realo, Voracek, and Allik (2008), so larger clinical samples should be investigated before drawing more definitive conclusions. Moreover, in future studies, using longitudinal data and age-balanced groups can allow for the investigation of developmental patterns, as described by Roberts and Del Vecchio (2000).

As a third major finding, the relations between the PID-5 higher order domains and the DAPP-BQ higher order dimensions generally mirror the nonclinical findings by Van den Broeck, Bastiaansen, Rossi, Dierckx, De Clercq, and Hofmans (2013) and further confirm the alignment of both models, with PID-5 Negative Affectivity, Detachment, and Antagonism showing strong and specific relations to DAPP-BQ Emotional Dysregulation, Inhibitedness, and Dissocial, respectively. Perhaps even more interesting however, differences between both models can be observed as well. First, the PID-5 Neuroticism Factor (Negative Affectivity) appears as far more specific than the DAPP-BQ Neuroticism Factor (Emotional Dysregulation); whereas PID-5 Neuroticism related strongly and specifically to DAPP-BQ Neuroticism, the latter correlated considerably with all but one (Antagonism) PID-5 higher order domains. Evidently, this difference in broadness (i.e., depending on the model used) can lead to different conclusions regarding relations between the latent variable (i.e., Neuroticism) and, for example, psychopathology. Second, although statistically significant, the size of the correlation between the PID-5 higher order domain Disinhibition and the DAPP-BQ higher 
order dimension Compulsivity suggests both constructs tap into considerably different contents. Moreover, PID-5 Disinhibition was substantially associated with DAPP-BQ Dissocial, whereas DAPP-BQ Compulsivity showed a nearzero correlation with PID-5 Antagonism. This matches the nonclinical findings of Van den Broeck, Bastiaansen, Rossi, Dierckx, De Clercq, and Hofmans (2013), describing also earlier controversy regarding the position of Disinhibition versus Compulsivity in Krueger et al. (2011). Whereas DAPP-BQ Compulsivity as a higher order dimension represents only one DAPP-BQ lower order trait (in fact, the lower order trait Compulsivity is the higher order dimension Compulsivity), referring to a tendency to be orderly and systematic and a preference for structure and organization, the (reversely keyed) PID-5 higher order domain Disinhibition could be understood as a broader concept. That is, PID-5 Disinhibition relates to Impulsivity, Irresponsibility, and Distractibility that could also be thought of as expressions of antisocial styles next to (the inverse of) intrapersonally and interpersonally moralistic motives through rule-bound behavior. Interestingly and in accordance with this, the DAPP-BQ higher order domain Compulsivity appears as a predictor (only) of the obsessive-compulsive personality disorder in Pukrop et al. (2009), whereas in Hopwood et al. (2012) the PID-5 higher order domain Disinhibition correlates considerably with the $D S M-I V$ antisocial personality disorder and close to zero with the obsessive-compulsive personality disorder. Further in line with our findings, the hierarchical analysis of PID-5 clinician ratings performed by Morey, Krueger, and Skodol (2013) identified a Compulsivity factor that only modestly related to the negative pole of the Disinhibition factor, suggesting that at least "clinicians conceptualize flexibility and adaptability —not impulsivity —as the inverse of Compulsivity" (Morey \& Skodol, 2013, p. 840). On an even more conceptual level, this finding also relates to the question of polarity, that is, the question whether both ends or only one end of a trait distribution are to be thought of as pathological (see Krueger \& Markon, 2014; Samuel, 2011, for an extensive discussion). Third, the PID-5 higher order domain Psychoticism, not separately represented in the four-factor DAPP-BQ model, related to DAPP-BQ Emotional Dysregulation, Inhibitedness, and Dissocial but also showed a considerable amount of variance not explained by the DAPP-BQ model (the lowest of all five PID-5 higher order domains, in fact). In other words, our results suggest that the PID-5 higher order domain Psychoticism shows incremental value as a "separate and additional spectrum to the integrative four-factor model" (Krueger \& Tackett, 2003, p. 123).

Important limitations to current study need to be addressed. Given that our purpose was to investigate the PID-5 higher order domain structure in a broadband inpatient sample, our factor analysis (exploratory structural equation modeling) was performed on the PID-5 primary facet scales, and not on the items. Although we first investigated whether the primary facet scales themselves were unidimensional, an item-based analysis (requiring a larger clinical sample) would, in future research, allow for even more definitive conclusions about the PID-5 factor structure in an entirely clinical context. Second, in our finding that patients diagnosed with a personality disorder differed on PID-5 scores from patients without a personality disorder, we did not account for the factor that actually determines personality disorder in the $D S M-5$ Section III conceptualization, namely Criterion A. Therefore, our comparison is by no means a conclusive test of discriminant validity. In fact, the personality traits formulated in Criterion B only account for style - be it a maladaptive one. The similar scores of patients with and patients without a $D S M-I V$ personality disorder on the PID-5 higher order domains Detachment as well as Psychoticism might be illustrative of this.

Although the original higher order factor structure was replicated, and relations between DAPP-BQ higher order dimensions and PID-5 higher order domains were both in line with theorized expectations as well as revealing important differences between both models, the use of more comprehensive criterion variables in future research might help us further advance our conceptual understanding of (pathological) personality. Given the recent work on the hierarchical relationship between one-, two-, three-, four-, and five-factor conceptualizations of personality structure (Morey et al., 2013; Wright \& Simms, 2014; Wright et al., 2012), this approach would be specifically interesting in relation to the clinical merits of a fifth higher order domain Psychoticism, that is, above and beyond clinical variance explained by pathological four-factor models like the one the DAPP-BQ (as a measure of it) stands for. More elaborate analyses within a clinical sample on primary facet scale level will be especially helpful to further clarify the one versus multidimensional nature of the higher order domain Psychoticism and disentangle its relationship not only with the Big-Five dimension Openness (see Chmielewski, Bagby, Markon, Ring, \& Ryder, 2014; De Fruyt et al., 2013; Piedmont, Sherman, \& Sherman, 2012; but also Ashton, Lee, de Vries, Hendrickse, \& Born, 2012; Gore \& Widiger, 2013), but with psychosis as a clinical syndrome (Tackett et al., 2008) and at-risk-mental-states (e.g., McGorry et al., 2009) as well.

Notwithstanding the limitations and suggestions for further research discussed above, the conclusions of current study confirm the PID-5 as a valid and reliable measure of personality dimensions applicable in clinical samples. Moreover, its psychometric properties are preserved in a non-English language, replicating the findings of De Fruyt et al. (2013) in their nonclinical Flemish sample. Of further interest specifically is the future analysis of the PID-5 higher order domain Psychoticism, which may be of particular value in the future research on psychosis that aspires to "broadening the scope of research to include a non-clinical psychosis phenotype" (Kelleher \& Cannon, 2011, p. 1). 


\section{Appendix}

\section{Higher Order Dimensions of DAPP-BQ and Higher Order Domains of PID-5 as Pathological Variants of the Five-Factor Model}

\begin{tabular}{llllll}
\hline Instrument & \multicolumn{1}{c}{ Factor I } & Factor II & Factor III & Factor IV \\
\hline NEO-PI & Neuroticism & Extraversion & Agreeableness & Conscientiousness & Openness \\
DAPP-BQ & Emotional Dysregulation & Inhibitedness & Dissocial & Compulsivity \\
PID-5 & Negative Affectivity & Detachment & Antagonism & Disinhibition & Psychoticism \\
\hline
\end{tabular}

Note. DAPP-BQ = Dimensional Assessment of Personality Pathology-Basic Questionnaire; PID-5 = Personality Inventory for DSM-5; NEO-PI = NEO Personality Inventory.

\section{Acknowledgments}

Special thanks to Leen Gielen and Mia Van Broekhoven, for their indispensable efforts in the process of data collection.

\section{Declaration of Conflicting Interests}

The author(s) declared no potential conflicts of interest with respect to the research, authorship, and/or publication of this article.

\section{Funding}

The author(s) received no financial support for the research, authorship, and/or publication of this article.

\section{Note}

1. Primary school completed $=6$ years; first half of secondary school completed $=9$ years; second half of secondary school completed $=12$ years; professional or academic bachelor's degree completed $=15$ years, master's degree completed $=$ 16.5 (as the duration of additional master's studies ranges between 1 and 2 years, depending on the field of study).

\section{References}

American Psychiatric Association. (2000). Diagnostic and statistical manual of mental disorders (4th ed.). Washington, DC: American Psychiatric Association.

American Psychiatric Association. (2013). Diagnostic and statistical manual of mental disorders (5th ed.). Washington, DC: American Psychiatric Association.

Anderson, J. L., Sellbom, M., Bagby, R. M., Quilty, L. C., Veltri, C. O. C., Markon, K. E., \& Krueger, R. F. (2013). On the convergence between PSY-5 domains and PID-5 domains and facets: Implications for Assessment of DSM-5 personality traits. Assessment, 20, 286-294. doi:10.1177/1073191112471141

Ashton, M. C., Lee, K., de Vries, R. E., Hendrickse, J., \& Born, M. (2012). The maladaptive personality traits of the personality inventory for $D S M-5$ (PID-5) in relation to the HEXACO personality factors and schizotypy/dissociation. Journal of Personality Disorders, 26, 641-659. doi:10.1521/ pedi.2012.26.5.641
Asparouhov, T., \& Muthén, B. (2009). Exploratory structural equation modeling. Structural Equation Modeling, 16, 397-438. doi:10.1080/10705510903008204

Bandalos, D. L. (2002). The Effects of Item Parceling on Goodness-of-Fit and Parameter Estimate Bias in Structural Equation Modeling. Structural Equation Modeling, 9, 78-102. doi:10.1207/S15328007SEM0901_5

Bandalos, D. L. (2008). Is parceling really necessary? A comparison of results from item parceling and categorical variable methodology. Structural Equation Modeling, 15, 211-240. doi:10.1080/10705510801922340

Beauducel, A., \& Yorck-Herzberg, P. (2006). On the performance of maximum likelihood versus means and variance adjusted weighted least squares estimation in CFA. Structural Equation Modeling, 13, 186-203. doi:10.1207/s15328007sem1302_2

Chmielewski, M., Bagby, M., Markon, K., Ring, A. J., \& Ryder, A. G. (2014). Openness to experience, intellect, schizotypal personality disorder, and psychoticism: Resolving the controversy. Journal of Personality Disorders, 28, 1-17. doi:10.1521/pedi_2014_28_128

Chmielewski, M., \& Watson, D. (2008). The heterogeneous structure of schizotypal personality disorder: Item-level factors of the schizotypal personality questionnaire and their associations with obsessive-compulsive disorder symptoms, dissociative tendencies, and normal personality. Journal of Abnormal Psychology, 117, 364-376. doi:10.1037/0021843X.117.2.364

Costa, P., Terracciano, A., \& McCrae, R. R. (2001). Gender differences in personality traits across cultures: Robust and surprising findings. Journal of Personality and Social Psychology, 81, 322-331. doi:10.1037/0022-3514.81.2.322

Costa, P. T., \& McCrae, R. R. (1992). The five-factor model of personality and its relevance to personality disorders. Journal of Personality Disorders, 6, 343-359. doi:10.1521/ pedi.1992.6.4.343

De Clercq, B., De Fruyt, F., Mervielde, I., Krueger, B., \& Markon, K. (2011). Official Dutch translation of the Personality Inventory for DSM-5 (PID-5). Unpublished manuscript.

De Fruyt, F., De Clercq, B., De Bolle, M., Wille, B., Markon, K., \& Krueger, R. F. (2013). General and maladaptive traits in a fivefactor framework for DSM-5 in a university student sample. Assessment, 20, 295-307. doi:10.1177/1073191113475808 
Donnellan, M. B., \& Lucas, R. E. (2008). Age differences in the big five across the life span: Evidence from two national samples. Psychology and Aging, 23, 558-566. doi:10.1037/ a0012897

Few, L. R., Miller, J. D., Rothbaum, A. O., Meller, S., Maples, J., Terry, D. P., . . Mackillop, J. (2013). Examination of the Section III DSM-5 diagnostic system for personality disorders in an outpatient clinical sample. Journal of Abnormal Psychology, 122, 1057-1069. doi:10.1037/a0034878

First, M. B., Gibbon, M., Spitzer, R. L., Williams, J. B. W., \& Benjamin, L. S. (1997). Structured clinical interview for DSM-IV Axis II disorders (SCID-II). Washington, DC: American Psychiatric Press.

Fossati, A., Krueger, R. F., Markon, K. E., Borroni, S., \& Maffei, C. (2013). Reliability and validity of the Personality Inventory for DSM-5 (PID-5): Predicting DSM-IV personality disorders and psychopathy in community-dwelling Italian adults. Assessment, 20, 689-708. doi:10.1177/1073191113504984

Gore, W. L., \& Widiger, T. A. (2013). The DSM-5 dimensional trait model and five-factor models of general personality. Journal of Abnormal Psychology, 122, 816-821.

Harkness, A. R., Finn, J. A., McNulty, J. L., \& Shields, S. M. (2012). The Personality Psychopathology-Five (PSY-5): Recent constructive replication and assessment literature review. Psychological Assessment, 24, 432-443. doi:10.1037/ a0025830

Harkness, A. R., McNulty, J. L., \& Ben-Porath, Y. S. (1995). The Personality Psychopathology Five (PSY-5): Constructs and MMPI-2 scales. Psychological Assessment, 7, 104-114. doi:10.1037/1040-3590.7.1.104

Hopwood, C. J., Thomas, K. M., Markon, K. E., Wright, A. G. C., \& Krueger, R. F. (2012). DSM-5 personality traits and DSM-IV personality disorders. Journal of Abnormal Psychology, 121, 424-432. doi:10.1037/a0026656

Hu, L., \& Bentler, P. M. (1998). Fit indexes in covariance structure modeling: Sensitivity to underparameterized model misspecification. Psychological Methods, 3, 424-453. doi:10.1037/1082-989X.3.4.424

Hu, L., \& Bentler, P. M. (1999). Cutoff criteria for fit indexes in covariance structure analysis: Conventional criteria versus new alternatives. Structural Equation Modeling, 6, 1-55. doi:10.1080/10705519909540118

Kelleher, I., \& Cannon, M. (2011). Psychotic-like experiences in the general population: Characterizing a high-risk group for psychosis. Psychological Medicine, 41, 1-6. doi:10.1017/ S0033291710001005

Kline, R. B. (2005). Principles and practices of structural equation modeling. New York, NY: Guilford.

Krueger, R. F., Eaton, N. R., Clark, L. A., Watson, D., Markon, K. E., Derringer, J., . . . Livesley, W. J. (2011). Deriving an empirical structure of personality pathology for DSM-5. Journal of Personality Disorders, 25, 170-191. doi:10.1521/ pedi.2011.25.2.170

Krueger, R. F., Derringer, J., Markon, K. E., Watson, D., \& Skodol, A. E. (2012). Initial construction of a maladaptive personality trait model and inventory for DSM-5. Psychological Medicine, 42, 1879-1890. doi:10.1017/S0033291711002674

Krueger, R. F., \& Markon, K. E. (2014). The role of the DSM-5 personality trait model in moving toward a quantitative and empirically based approach to classifying personality and psychopathology. Annual Review of Clinical Psychology, 10, 477-501. doi:10.1146/annurev-clinpsy-032813-153732

Krueger, R. F., \& Tackett, J. L. (2003). Personality and psychopathology: Working toward the bigger picture. Journal of Personality Disorders, 17, 109-128. doi:10.1521/ pedi.17.2.109.23986

Livesley, W. J., \& Jackson, D. N. (2009). Manual for the Dimensional Assessment of Personality Pathology-Basic Questionnaire (DAPP-BQ). Port Huron, MI: Sigma Press.

McGorry, P., Nelson, B., Amminger, G. P., Bechdolf, A., Francey, S. M., Berger, G., . . Y Yung, A. R. (2009). Intervention in individuals at ultra-high risk for psychosis: A review and future directions. Journal of Clinical Psychiatry, 70, 1206-1213.

McLean, C. P., \& Anderson, E. R. (2009). Brave men and timid women? A review of the gender differences in fear and anxiety. Clinical Psychology Review, 29, 496-505. doi:10.1016/j. cpr.2009.05.003

Morey, L. C., Krueger, R. F., \& Skodol, A. E. (2013). The hierarchical structure of clinician ratings of proposed DSM-5 pathological personality traits. Journal of Abnormal Psychology, 122, 836-841. doi:10.1037/a0034003

Muthén, L. K., \& Muthén, B. O. (1998-2012). Mplus user's guide (7th ed.). Los Angeles, CA: Author.

Piedmont, R. L., Sherman, M. F., \& Sherman, N. C. (2012). Maladaptively high and low openness: The case for experiential permeability. Journal of Personality, 80, 1641-1668. doi: $10.1111 /$ j.1467-6494.2012.00777

Pukrop, R., Steinbring, I., Gentil, I., Schulte, C., Larstone, R., \& Livesley, J. W. (2009). Clinical validity of the dimensional assessment of personality pathology (DAPP) for psychiatric patients with and without a personality disorder diagnosis. Journal of Personality Disorders, 23, 572-586. doi:10.1521/ pedi.2009.23.6.572

Quilty, L. C., Ayearst, L., Chmielewski, M., Pollock, B. G., \& Bagby, R. M. (2013). The psychometric properties of the Personality Inventory for $D S M-5$ in an APA DSM-5 Field Trial sample. Assessment, 20, 362-369. doi:10.1177/1073191113486183

Roberts, B. W., \& Del Vecchio, W. F. (2000). The rank-order consistency of personality traits from childhood to old age: A quantitative review of longitudinal studies. Psychological Bulletin, 126, 3-25. doi:10.1037//0033-2909.126.1.3

Ryder, A. G., Costa, P. T., \& Bagby, M. (2007). Evaluation of the SCID II Personality Disorder Traits for DSM-IV: Coherence, discrimination, relations with general personality traits, and functional impairment. Journal of Personality Disorders, 21, 626-637. doi:10.1521/pedi.2007.21.6.626

Samuel, D. B. (2011). Assessing personality in the DSM-5: The utility of bipolar constructs. Journal of Personality Assessment, 93, 390-397. doi:10.1080/00223891.2011.57 7476

Samuel, D. B., Hopwood, C. J., Krueger, R. F., Thomas, K. M., \& Ruggero, C. J. (2013). Comparing methods for scoring personality disorder types using maladaptive traits in DSM5. Assessment, 20, 353-361. doi:10.1177/1073191113486182

Schmitt, D. P., Realo, A., Voracek, M., \& Allik, J. (2008). Why can't a man be more like a woman? Sex differences in Big Five Personality traits across 55 cultures. Journal of Personality 
and Social Psychology, 94, 168-182. doi:10.1037/00223514.94.1.168

Tackett, J. L., Silberschmidt, A. L., Krueger, R. F., \& Sponheim, S. R. (2008). A dimensional model of personality disorder: Incorporating DSM cluster A characteristics. Journal of Abnormal Psychology, 117, 454-459. doi:10.1037/19492715.S.1.27

Thomas, K. M., Yalch, M. M., Krueger, R. F., Wright, A. G. C., Markon, K. E., \& Hopwood, C. J. (2012). The convergent structure of $D S M-5$ personality trait facets and five-factor model trait domains. Assessment, 20, 308-311. doi: $10.1177 / 1073191112457589$

Trull, T., \& Durrett, C. A. (2005). Categorical and dimensional models of personality disorder. Annual Review of Clinical Psychology, 1, 355-380. doi:10.1146/annurev.clinpsy.1.102803. 144009

Van den Broeck, J., Bastiaansen, L., Rossi, G., Dierckx, E., \& De Clercq, B. (2013). Age-neutrality of the trait facets proposed for personality disorders in DSM-5: A DIFAS analysis of the PID-5. Journal of Psychopathology and Behavioral Assessment, 35, 487-494. doi:10.1007/s10862-013-9364-3

Van den Broeck, J., Bastiaansen, L., Rossi, G., Dierckx, E., De Clercq, B., \& Hofmans, J. (2013). Hierarchical structure of maladaptive personality traits in older adults: Joint factor analysis of the PID-5 and the DAPP-BQ. Journal of Personality Disorders, 28, 198-211. doi:10.1521/pedi $201327 \quad 114$
Van Kampen, D., \& De Beurs, E. (2009). DAPP-BQ. Dimensionale assessment van persoonlijkheidspathologie inclusief screeningsversie. Amsterdam, The Netherlands: Hogreve Uitgevers B.V.

Watson, D., Stasik, S. M., Ro, E., \& Clark, L. A. (2013). Integrating normal and pathological personality: Relating the DSM5 trait-dimensional model to general traits of personality. Assessment, 20, 312-326. doi:10.1177/1073191113485810

Widiger, T. A., \& Simonsen, E. (2005). Alternative dimensional models of personality disorder: Finding common ground. Journal of Personality Disorders, 19, 110-130. doi:10.1521/ pedi.19.2.110.62628

Wright, A. G. C., \& Simms, L. J. (2014). On the structure of personality disorder traits: Conjoint analyses of the CAT-PD, PID-5, and NEO-PI-3 trait models. Personality Disorders, 5 , 43-54. doi:10.1037/per0000037

Wright, A. G. C., Thomas, K. M., Hopwood, C. J., Markon, K. E., Pincus, A. L., \& Krueger, R. F. (2012). The hierarchical structure of DSM-5 pathological personality traits. Journal of Abnormal Psychology, 121, 951-957. doi:10.1037/a0027669

Zimmerman, J., Altenstein, D., Krieger, T., Holtforth, M. G., Pretsch, J., Alexopoulos, J., . . L Leising, D. (2014). The structure and correlates of self-reported DSM-5 maladaptive personality traits: Findings from two German-speaking samples. Journal of Personality Disorders, 28, 1-23. doi:10.1521/ pedi_2014_28_130 $\alpha \alpha \alpha$ 